University of Nebraska - Lincoln

DigitalCommons@University of Nebraska - Lincoln

$11-1-2000$

\title{
Comparative studies of dissociative electron attachment to methyl halides
}

\author{
R. S. Wilde \\ University of Nebraska-Lincoln \\ Gordon A. Gallup \\ University of Nebraska-Lincoln, ggallup1@unl.edu \\ Ilya I. Fabrikant \\ University of Nebraska-Lincoln, ifabrikant@unl.edu
}

Follow this and additional works at: https://digitalcommons.unl.edu/physicsgallup

Part of the Physics Commons

Wilde, R. S.; Gallup, Gordon A.; and Fabrikant, llya I., "Comparative studies of dissociative electron attachment to methyl halides" (2000). Gordon Gallup Publications. 48.

https://digitalcommons.unl.edu/physicsgallup/48

This Article is brought to you for free and open access by the Research Papers in Physics and Astronomy at DigitalCommons@University of Nebraska - Lincoln. It has been accepted for inclusion in Gordon Gallup Publications by an authorized administrator of DigitalCommons@University of Nebraska - Lincoln. 


\title{
Comparative studies of dissociative electron attachment to methyl halides
}

\author{
R. S. Wilde, G. A. Gallup, and I. I. Fabrikant \\ Department of Physics and Astronomy, \\ University of Nebraska-Lincoln, Lincoln, NE 68588-0111, USA
}

Submitted June 2000

\begin{abstract}
The dissociative electron attachment cross sections for the methyl halides vary in an enormous range from the virtually unmeasurable $10^{-23} \mathrm{~cm}^{2}$ for $\mathrm{CH}_{3} \mathrm{Cl}$ at room temperature to $10^{-14} \mathrm{~cm}^{2}$ for $\mathrm{CH}_{3} \mathrm{I}$. In this paper we supplement our previous studies by calculations of dissociative electron attachment to $\mathrm{CH}_{3} \mathrm{Br}$ and compare results for all methyl halides studied so far. The rate as a function of temperature for $\mathrm{CH}_{3} \mathrm{Cl}$ and $\mathrm{CH}_{3} \mathrm{Br}$ exhibits an exponential dependence on $1 / T$ (Arrhenius law) with the activation energy lower for $\mathrm{CH}_{3} \mathrm{Br} \mathrm{CH}_{3} \mathrm{I}$ does not obey the Arrhenius law since the crossing point of the neutral and anion potential curves occurs near the lowest vibrational levels. The cross section as a function of electron energy for all of the methyl halides studied here exhibits structure at the vibrational excitation thresholds that is associated with a vibrational Feshbach resonance like that observed recently in $\mathrm{CH}_{3} \mathrm{I}$ by Schramm et al (Schramm A, Fabrikant I I, Weber J M, Leber E, Ruf M-W and Hotop H 1999 J. Phys. B: At. Mol. Opt. Phys. 32 2153).
\end{abstract}

\section{Introduction}

Inelastic collisions between low-energy electrons and methyl halide molecules may be modeled by assuming that only one vibrational mode is excited by the collision. This mode corresponds to the symmetric $\mathrm{C}-\mathrm{X}$ stretch in the molecule $\mathrm{CH}_{3} \mathrm{X}$, where $\mathrm{X}$ stands for an $\mathrm{F}, \mathrm{Cl}, \mathrm{Br}$ or I atom. A comparative study of dissociative electron attachment (DEA) to these molecules can help understand the basic physics governing the magnitudes of the cross sections and attachment rates for different molecules and the dependence of the cross section on vibrational temperature and electron energy.

Several experimental and theoretical studies of DEA have been performed for the methyl halides. DEA to $\mathrm{CH}_{3} \mathrm{Cl}$ was measured by several experimental groups [1-4] using both swarm and beam techniques with very different results, that disagree by many orders of magnitude. Elaborate experimental investigations $[5,6]$, supported by semi-empirical calculations [6-8], showed that most of these measurements were affected by contaminants, and the actual value of the DEA cross section for $\mathrm{CH}_{3} \mathrm{Cl}$ at room temperature is so small that the process can hardly be detected. However, the increased temperature leads to a very rapid exponential increase of the cross section. The theory and experiment agree very well starting from the temperature of about $500 \mathrm{~K} \mathrm{[6].}$

DEA cross sections for $\mathrm{CH}_{3} \mathrm{Br}$ are substantially larger than for $\mathrm{CH}_{3} \mathrm{Cl}$. The swarm-unfolded DEA cross section of [9] shows a room-temperature peak at $0.38 \mathrm{eV}$ with a peak magnitude of about $1.8 \times 10^{-18} \mathrm{~cm}^{2}$. Several measurements of the electron attachment rate constant for $\mathrm{CH}_{3} \mathrm{Br}$ have also been made in the past 30 years. The rate constant near room temperature has been measured in [9-15], using various experimental techniques, to be between 3.6 and $10.8 \times 10^{-12} \mathrm{~cm}^{3} \mathrm{~s}^{-1}$. 
In the past there was some discrepancy between the swarm measurements of the attachment rate $[17,18]$ and other measurements. In [9] it was shown that the very large attachment rates of [17, 18] could not be due to $\mathrm{CH}_{3} \mathrm{Br}$ and that the sample must have been affected by some contaminant. The energy integrated DEA cross sections were also measured in an electron beam experiment by Spence and Schulz [19]. Both the energy integrated cross sections and the attachment rate for $\mathrm{CH}-$ ${ }_{3} \mathrm{Br}$ exhibit a very dramatic increase with increasing vibrational temperature $[9-11,19]$.

DEA to $\mathrm{CH}_{3} \mathrm{I}$ was studied experimentally by electron beam [19] and swarm [11, 20-22] methods and using the threshold photoelectron attachment technique [23]. In recent studies of DEA to the $\mathrm{CH}_{3} \mathrm{I}$ molecule $[24,25]$ performed with a very high-energy resolution, a sharp variation of the cross section within a narrow energy interval below the first threshold for vibrational excitation of the symmetric stretch was observed. This was interpreted [24] in terms of a vibrational ("nuclearexcited' [26, 27]) Feshbach resonance. Higher vibrational thresholds are free of the resonance but exhibit pronounced cusps of the Baz'-Wigner type [28].

The goal of this paper is to supplement our previous studies by theoretical calculations of DEA to $\mathrm{CH}_{3} \mathrm{Br}$ and compare results for all methyl halides studied so far. In particular we are interested in the physical mechanisms responsible for the large difference of the DEA cross sections corresponding to different compounds and in comparing the temperature and energy dependence of the rates. Another question is related to the vibrational Feshbach resonance: is this something typical for $\mathrm{CH}_{3} \mathrm{I}$ or does it appear in all other methyl halides? Our previous results for $\mathrm{CH}_{3} \mathrm{Cl}[7,8]$ show structure in the temperature-averaged cross sections. We show in this paper that this structure can be connected with vibrational Feshbach resonances in the individual (in vibrational quantum number $v$ ) cross sections. However, the observation of these resonances is possible only in experiments with very highenergy resolution, which has been achieved only in the measurements of DEA to $\mathrm{CH}_{3} \mathrm{I}[24,25]$.

The rest of the paper is organized as follows. We describe our procedure for calculating DEA to $\mathrm{CH}_{3} \mathrm{Br}$ and compare our results with available experimental data in section 2 . Then we compare the energy and temperature dependence of the cross sections for different halides in section 3 . We note that we have not considered $\mathrm{CH}_{3} \mathrm{~F}$ here. The vertical attachment energy for $\mathrm{CH}_{3} \mathrm{~F}$ has been estimated from electron transmission spectroscopy (ETS) to be nearly $5 \mathrm{eV}$ [29] and it might be assumed from this that the DEA cross section for $\mathrm{CH}_{3} \mathrm{~F}$ is even smaller than that of $\mathrm{CH}_{3} \mathrm{Cl}$. In section 4 we discuss the vibrational Feshbach resonances for all methyl halides. Section 5 is a brief summary of our main conclusions.

\section{Theory for methyl bromide}

\subsection{Models}

Previously we have used the semi-empirical $R$-matrix theory to calculate vibrational excitation (VE) and DEA cross sections for $\mathrm{CH}_{3} \mathrm{Cl}[7,8]$ and for $\mathrm{CF}_{3} \mathrm{Cl}$ [30]. There, experimental data on the VE process were used to obtain the parameters of the fixed nucleus $R$-matrix in the single-pole approximation

$$
R(\rho)=\frac{\gamma^{2}(\rho)}{W(\rho)-E_{\mathrm{e}}}+R_{\mathrm{b}}
$$

where $W(\rho)$ is the $R$-matrix eigenvalue, $E_{\mathrm{e}}$ the electron energy, $\rho$ the internuclear separation relative to equilibrium and $R_{\mathrm{b}}$ a background term, which is assumed to be independent of $E_{\mathrm{e}}$ and $\rho$. The reduced width $\gamma(\rho)$ has been parametrized before as $[7,8,30]$

$$
\gamma(\rho)=\gamma_{0}+\frac{\gamma_{1}}{\mathrm{e}^{\zeta \rho}+\eta} .
$$


Table 1. Parameters of the $R$-matrix model for the methyl halides studied here.

\begin{tabular}{llllllll}
\hline & $r_{0}$ & $\gamma_{0}$ & $\gamma_{1}$ & $\zeta$ & $\eta$ & $R_{\mathrm{b}}$ & $\beta$ \\
\hline $\mathrm{CH}_{3}$ Br model 1 & 5.0 & 0.0 & 1.406 & 4.581 & 0.3145 & 0.1588 & 1.437 \\
$\mathrm{CH}_{3} \mathrm{Br}$ model 2 & 5.0 & 0.0 & 1.50 & 1.42 & 2.643 & 0.072 & 1.3753 \\
$\mathrm{CH}_{3} \mathrm{CI}$ & 4.0 & 0.154 & 0.227 & 1.65 & 0.824 & 0.3 & 0.7297 \\
$\mathrm{CH}_{3} \mathrm{I}$ & 5.0 & 0.0 & 0.4902 & 1.022 & 0.131 & 0.9076 & 1.996 \\
\hline
\end{tabular}

Other input parameters of the $R$-matrix theory include the potential curve of the neutral molecule, parametrized in the form of a Morse function,

$$
V(\rho)=A\left(\mathrm{e}^{-\alpha \rho}-1\right)^{2},
$$

and the potential curve for the temporary negative-ion state, which we parametrize in the generalized Morse form

$$
U(\rho)=B \mathrm{e}^{-2 \beta \rho}-C \mathrm{e}^{-\beta \rho}+D .
$$

This potential curve is related to the $R$-matrix pole, $W(\rho)$, by

$$
W(\rho)=U(\rho)-V(\rho) .
$$

We note that the $R$-matrix definition of the diabatic curve is different from that used in the projection operator theory [31]. In particular, the shift,

$$
\Delta(\rho)=U_{\text {ad }}(\rho)-U(\rho),
$$

is not negatively defined in the $R$-matrix theory. Here, $U_{\text {ad }}(\rho)$ is the adiabatic negative-ion potential curve.

For the case of $\mathrm{CH}_{3} \mathrm{Br}$, the parameters $\gamma_{0}$ and $C$ have been set equal to zero in order to reduce the number of fit parameters and to simplify the model of the diabatic negative-ion curve. For the neutral curve, $A$ and $\alpha$ were chosen to give the empirical values for the dissociation energy of $\mathrm{CH}_{3} \mathrm{Br}, 3.078 \mathrm{eV}$ [9], as well as the vibrational ( $v=0-1)$ spacing of $75.8 \mathrm{meV}$ [32]. For the negative-ion curve, we have fixed the vertical attachment energy to the electron transmission spectra estimate of [29] of $2.4 \mathrm{eV}$ ( $B$ $=0.09754 \mathrm{au})$ and the asymptotic value using the electron affinity of $\mathrm{Br}, 3.37 \mathrm{eV}[3](D=-0.00934$ $\mathrm{au}$ ). This leaves the parameter $\beta$ undetermined and, as done previously for $\mathrm{CH}_{3} \mathrm{I}$, it is used as a fit parameter. To our knowledge there is no measurement of $\mathrm{VE}$ for $\mathrm{CH}_{3} \mathrm{Br}$ in the literature, so our previous method of fitting the parameters of the $R$-matrix to VE cross sections and then using the obtained parameters to calculate the DEA cross sections cannot be employed here. Instead we have tried to fit the temperature-averaged DEA cross section at $300 \mathrm{~K}$ to the swarm-unfolded cross sections of Datskos et al [9] in the region of the broad peak centered near $0.38 \mathrm{eV}$, then the DEA cross sections can be calculated using these parameters and the calculated temperature dependence can be compared with the experimental measurements. Hereafter we refer to this procedure as "model 1." The obtained fit parameters are given in Table 1. The value of the reduced width at the equilibrium internuclear separation in this case is very large, as compared with the other methyl halides, and is thus perhaps unrealistic. Also, the attachment rate constant we calculate using model 1 seems too small (see next section).

We have also fitted the adiabatic negative-ion potential curve to ab initio calculations of the curve, at the second-order Möller-Plesset (MP2) ab initio level (described in more detail in the next paragraph), for the region $\rho>\rho_{\mathrm{c}}$, by varying the parameters of $\gamma(\rho)$. We note that the value of $\beta$ was adjusted to fit $U(\rho)$ to the first MP2 point to the left of the crossing point $\rho_{\mathrm{c}}$. The parameters obtained in this way correspond to model 2 of Table 1 . The diabatic and adiabatic potential curves for these "model 2" parameters are shown in Figure 1. The adiabatic potential curve may be calcu- 


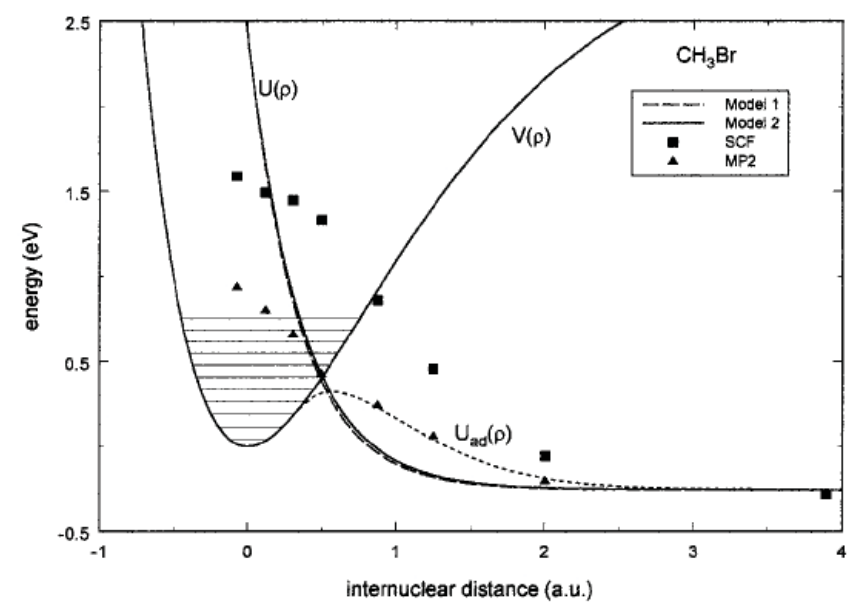

Figure 1. Potential curves for $\mathrm{CH}_{3} \mathrm{Br} . V(\rho)$ is the neutral curve and $U(\rho)$ is the anion curve. We also show the $a b$ initio SCF and MP2 calculations of the anion curve which are described in the text. The lowest vibrational levels are also shown.

lated by solving the $R$-matrix matching equation for the electron energy in the region $\rho>\rho_{\mathrm{c}}$ as in [7, 8]. We have not shown the adiabatic curve calculated from the model 1 parameters, because it is essentially the same as the diabatic curve. This is due to the small value of the reduced width, $\gamma(\rho)$, for $\rho>\rho_{\mathrm{c}}$.

The calculations to obtain the negative-ion curve were carried out using the GAMESS [33] computation package. The basis used was Huzinaga's 21-split-valence-orbital set [34] (called "midi" in GAMESS) augmented with standard d and diffuse sp sets. Electron correlation was introduced using the MP2 approach. In line with the other semi-phenomenological parts of this theory, we adjusted the curve so the asymptotic energy was consistent with the experimental value of the electron affinity of Br. In Figure 1 we show the separately shifted curves from the restricted open-shell Hartree-Fock calculations (these points are labeled SCF for self-consistent field in Figure 1) and after the subsequent MP2 corrections. The principal physical effect expected from the MP2 correction is the inclusion of terms contributing to van der Waals forces between the methyl group and the $\mathrm{Br}^{-}$ion.

\subsection{Results}

The attachment rates $k_{\text {att }}$, at a particular electron temperature, can be calculated by averaging our DEA cross sections $\sigma_{\mathrm{DEA}}$ over the Maxwell velocity distribution for the attaching electron,

$$
k_{\mathrm{att}}(T)=\frac{2^{3 / 2}}{m_{\mathrm{e}}^{1 / 2} \pi^{1 / 2} T^{3 / 2}} \int_{0}^{\infty} E \sigma_{\mathrm{DEA}}(E, T) \exp \left(-\frac{E}{T}\right) \mathrm{d} E,
$$

where $m_{\mathrm{e}}$ is the electron mass and the temperature, $T$, is in energy units. Here we assume the vibrational and electron temperatures are the same. For the model 1 parameters the calculated $k_{\text {att }}(T$ $=300 \mathrm{~K}$ ) is $1.60 \times 10^{-12} \mathrm{~cm}^{3} \mathrm{~s}^{-1}$. This value is substantially lower than the measured value of [9], which is $10.8 \times 10^{-12} \mathrm{~cm}^{3} \mathrm{~s}^{-1}$. The discrepancy may be due to the fact that in [9] the values of the attachment cross section are not shown for electron energies below about $0.15 \mathrm{eV}$. Due to the decreasing exponential term, $\mathrm{e}^{-E / T}$, in equation (7) and the zero-energy peak in the DEA cross section, it is the cross section at lower electron energies which is most important for the evaluation of the 


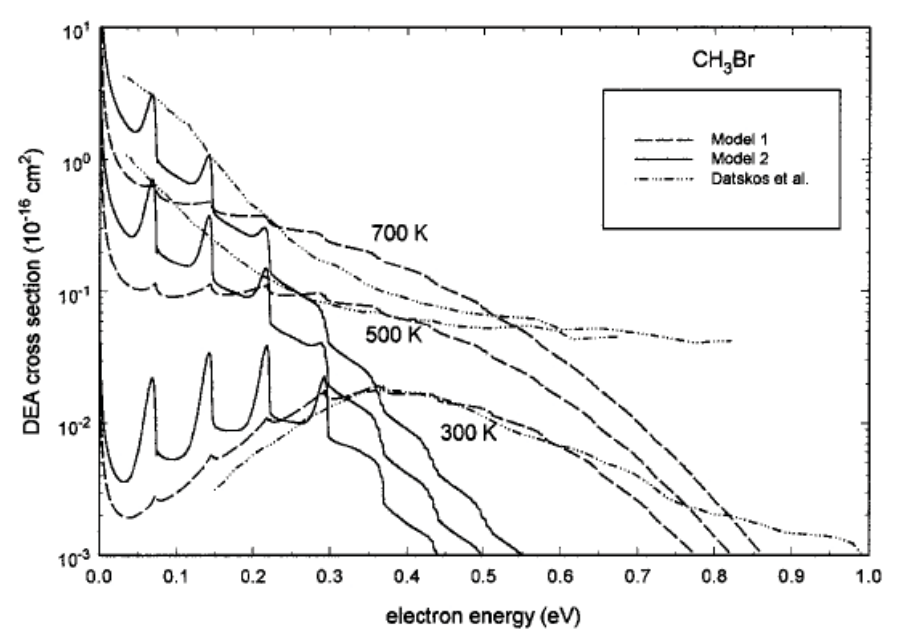

Figure 2. Temperature dependent DEA cross sections at $T=300,500$ and $700 \mathrm{~K}$. Dashed curve: calculations using parameters of model 1. Solid curve: calculations using parameters of model 2. Chain curve: swarm-unfolded cross section of Datskos et al [9].

thermal electron attachment rates. In order for the attachment rate to be as large as the measurement of [9] the attachment cross section would have to exhibit an increase for these low electron energies.

In Figure 2 we show the temperature dependence of the calculated DEA cross sections for temperatures 300, 500 and $700 \mathrm{~K}$ along with the swarm-unfolded cross sections [9] at these temperatures. As was mentioned above, the model 2 parameters were fitted to the ab initio calculations at the MP2 level. This has had the effect of increasing the cross sections at lower energies; however, the broad peak near $0.38 \mathrm{eV}$ has disappeared. The crossing points for the two diabatic curves are very similar and the changes in the DEA cross section seem to be mainly due to the difference in the reduced width function between the two models. The swarm cross sections near $0 \mathrm{eV}$ increase more quickly with increasing vibrational temperature than do our calculations for either model 1 or model 2. This is in spite of the fact that the DEA cross sections from model 2 are substantially larger than the swarm measurements at room temperature. This, along with the fact, mentioned previously, that our attachment rate derived from the model 1 cross section at $T=300 \mathrm{~K}$ using equation (7) is smaller than the reported value of [9], might suggest that the swarm measurements are still not entirely due to only $\mathrm{CH}_{3} \mathrm{Br}$ at very low electron energies, but may still contain some contaminant which affects the measurements at low electron energies, or that the swarm-unfolding procedure is not able to properly take into account the structure in the DEA cross section at low energies. It is likely that only high-resolution experiments, such as the laser photoelectron attachment of [24, 25], can accurately determine the structure of the low-energy attachment cross section. As we have seen, this structure can have a substantial effect on the attachment rate coefficient.

The diabatic negative-ion curves are only slightly different comparing model 1 with model 2. The adiabatic curves in the region $\rho>\rho_{c}$ are quite different, however. We show in Figure 3 DEA cross sections from the lowest individual vibrational levels of $\mathrm{CH}_{3} \mathrm{Br}$. The increase of the reduced width near the crossing point, from model 1 to model 2, acts to increase the individual DEA cross sections at low electron energies. The structure near the $v=4$ threshold especially increases. This shows that the threshold structure is very sensitive to the adiabatic curve, through the increase of 

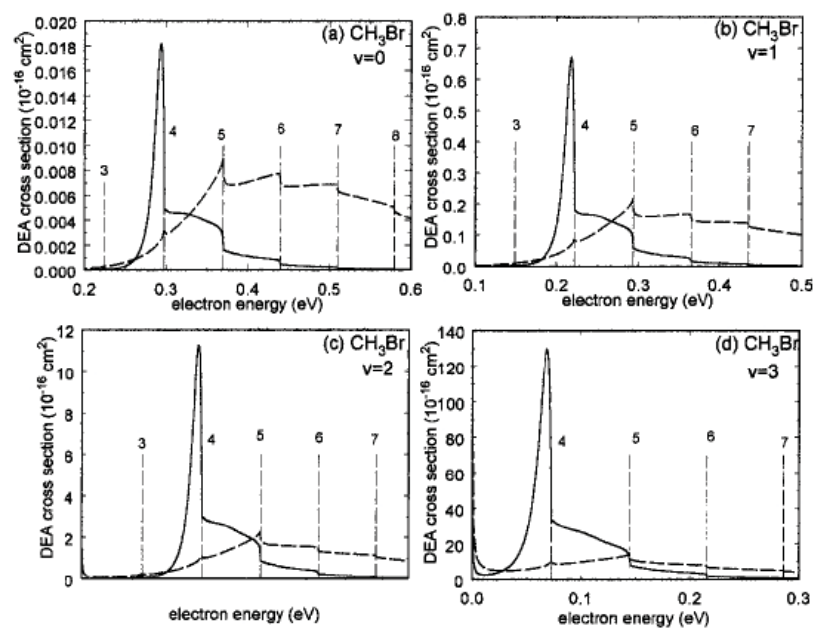

Figure 3. DEA cross sections from the individual vibrational levels, $v=0,1,2$ and 3 , of $\mathrm{CH}_{3} \mathrm{Br}$. Solid curve: model 1. Dashed curve: model 2. Vertical dashed lines denote the indicated vibrational thresholds.

the reduced width. The threshold structure is connected with the vibrational Feshbach resonance to be discussed further in section 4 . We also note that the single resonance feature in the individual cross sections is responsible for the several peaks seen in the temperature-averaged DEA cross section, due to its appearance at different energies in the cross sections from different initial vibrational levels.

In Figure 4 we show the temperature dependence of the attachment rates calculated from equation (7) for $\mathrm{CH}_{3} \mathrm{Br}$ compared with the measurements of $[9-11,16]$. To calculate the attachment rates from the calculated DEA cross sections the integration in equation (7) must be carried out. Since the methyl halides have dipole moments which are super-critical or very near the critical value $\left(\mu_{\mathrm{cr}}=0.6395 \mathrm{au}\right)$ [35] we assume the cross section has the form $\sigma_{\mathrm{DEA}}\left(E_{\mathrm{e}}, T\right) \rightarrow$ const $\times E_{\mathrm{e}}^{-x}$ as the electron energy $E_{\mathrm{e}} \rightarrow 0$. The constant and $x$ are adjusted to fit our calculated cross sections out to about $E_{\mathrm{e}}=3 \mathrm{meV}$, where $x$ is close to 1 .

We see from Figure 4 that the attachment rates behave as a function of temperature according to the familiar Arrhenius type equation

$$
k_{\text {att }}(T)=C(T) \mathrm{e}^{-\frac{E_{3}}{T}} .
$$

The constant $E_{\mathrm{a}}$ is known as the activation energy. For model 1 we obtain $E_{\mathrm{a}}=257 \mathrm{meV}$ and for model $2 E_{\mathrm{a}}=249 \mathrm{meV}$. This shows that the activation energy is not as sensitive to the reduced width as it is to the location of the crossing point. We will see in some more detail why this is in section 5 . Since the crossing point is very nearly the same for models 1 and 2 the activation energies are quite similar. Experimental values for the activation energy are $260 \pm 15 \mathrm{meV}$ [10], $247 \pm$ $17 \mathrm{meV}$ [14] and $300 \mathrm{meV}$ [11].

It is clear that the $R$-matrix method used here can reproduce the values of attachment rates and activation energy seen experimentally if the negative-ion potential curve and the reduced width are known accurately near the crossing point, $\rho_{\mathrm{c}}$. Small adjustments of these parameters can cause large differences in these quantities. The shape of the DEA cross section for low electron energies is very dependent on both the adiabatic curve and the reduced width while the temperature depen- 


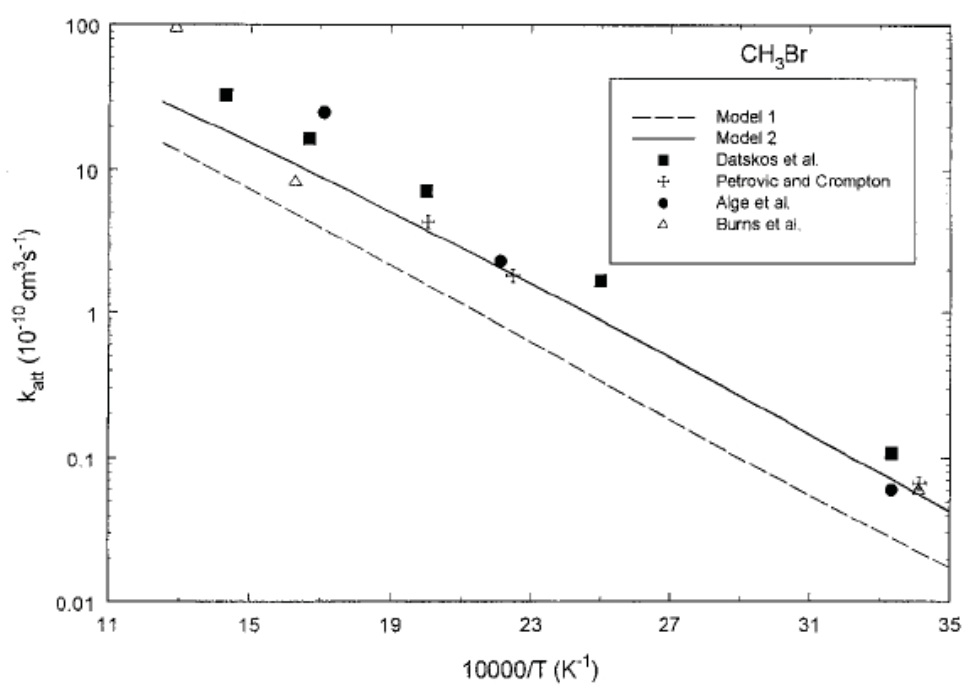

Figure 4. Arrhenius type plot of the calculated attachment rates, $k_{\text {att }}$, for $\mathrm{CH}_{3} \mathrm{Br}$ compared with several experimental measurements, Datskos et al [9], Petrovic and Crompton [10], Alge et al [11] and Burns et al [16].

dence and magnitudes of the DEA cross section and attachment rates are mainly dependent upon the location of the crossing point of the neutral and diabatic negative-ion potential curves.

\section{General discussion of DEA to methyl halides}

In the present calculations for $\mathrm{CH}_{3} \mathrm{Cl}$ we use a set of parameters shown in Table 1, which is taken from the previous calculations of Fabrikant [8]. These calculations provide good agreement with the measured temperature dependence of Pearl et al [6] for temperatures above about $500 \mathrm{~K}$. The parameters for the negative-ion curve and reduced width were chosen so as to agree with the calculations of the adiabatic curve in the region $\rho>\rho_{\mathrm{c}}$ of Falcetta and Jordan [36]. The parameters for $\rho<\rho_{\mathrm{c}}$ were chosen to reproduce the observed value of the vertical attachment energy [37] and the features observed in the experimental measurements of the differential VE cross section at a scattering angle of $100^{\circ}[38]$.

The previous calculations using the present $R$-matrix method for $\mathrm{CH}_{3} \mathrm{Cl}[7,8]$ both show structure near each vibrational threshold in the DEA cross sections. We now calculate the DEA cross sections for $\mathrm{CH}_{3} \mathrm{Cl}$ using a finer energy step. The parameters used for $\mathrm{CH}_{3} \mathrm{I}$ are from [24] and are also displayed in Table 1.

The potential curves employed in the present calculations for $\mathrm{CH}_{3} \mathrm{Cl}$ and $\mathrm{CH}_{3} \mathrm{I}$ are shown in Figure 5. We emphasize that the parameters for $\mathrm{CH}_{3} \mathrm{I}$ were obtained by fitting to the high-resolution measurements in [24] in the region of the pronounced vibrational Feshbach resonance below the $v=1$ threshold. Again we see a large difference between the SCF and MP2 calculations of the negative-ion curve for $\mathrm{CH}_{3}$ I. There is also fair agreement between the MP2 curve and the adiabatic curve obtained from the $R$-matrix parameters.

In Figure 6 we compare the temperature-averaged DEA cross sections for the methyl halides at room temperature, $T=300 \mathrm{~K}$. We show calculations for both models 1 and 2 for $\mathrm{CH}_{3} \mathrm{Br}$. The magnitudes of the cross sections vary over a very large range at low electron energies. As was seen 

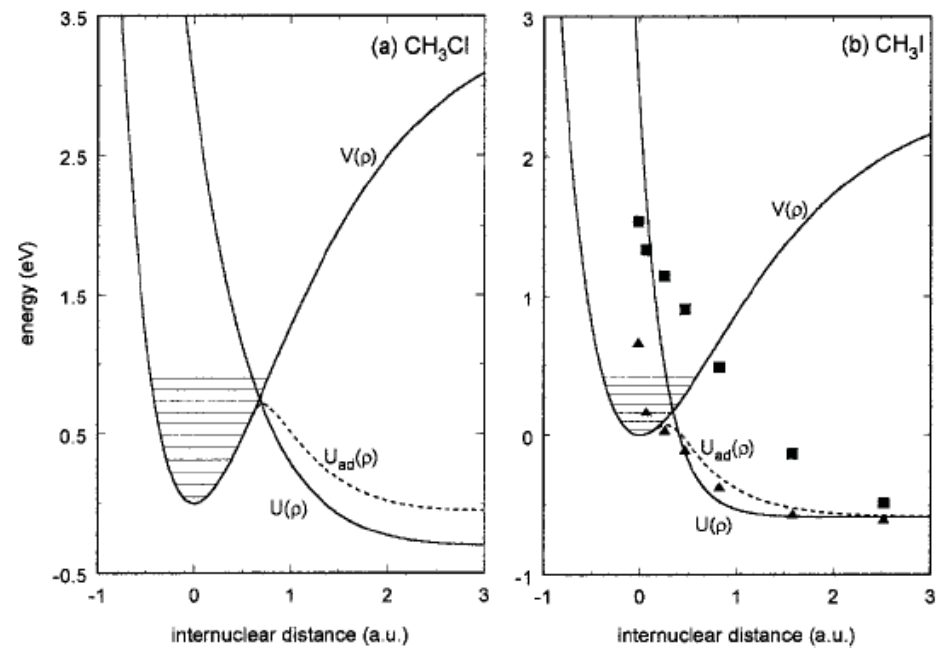

Figure 5. Potential curves for $\mathrm{CH}_{3} \mathrm{Cl}$ and $\mathrm{CH}_{3} \mathrm{I}$. The lowest vibrational levels are shown as the short-dashed lines.

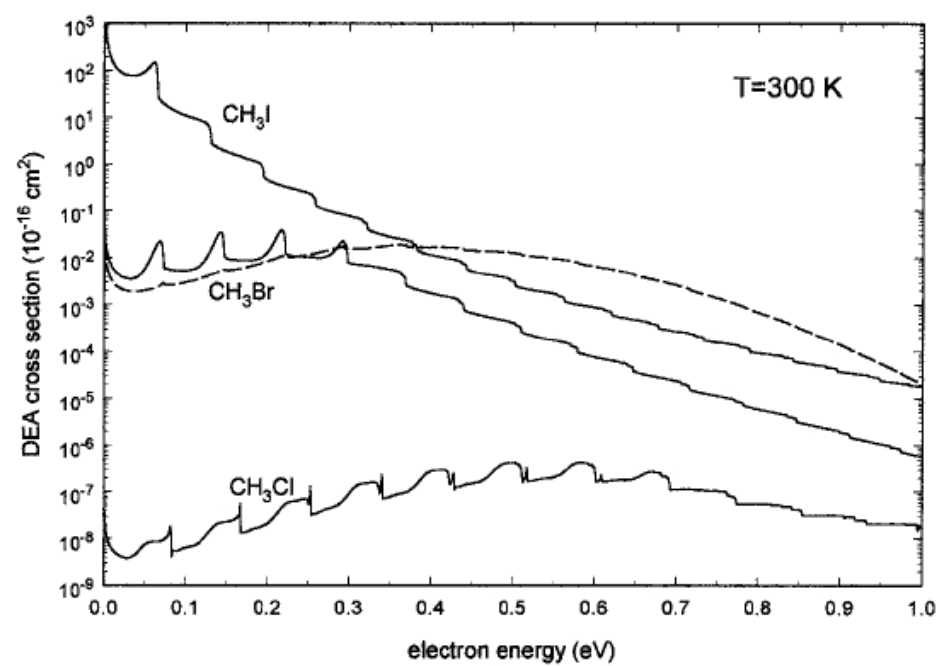

Figure 6. DEA cross sections at room temperature, $T=300 \mathrm{~K}$, for all methyl halides.

previously for the two models describing $\mathrm{CH}_{3} \mathrm{Br}$ this is due mainly to the difference in the position of the crossing point of the neutral and anion potential curves in each methyl halide. Comparing the potential curves for the various molecules of Figures 1 and 5 we see that as the crossing point moves to lower energy the DEA cross sections become larger. The structures, similar to that seen in $\mathrm{CH}_{3} \mathrm{I}$ [24], near each vibrational threshold can now be connected with vibrational Feshbach resonances in each molecule. For $\mathrm{CH}_{3} \mathrm{Br}$, the structures are more pronounced for model 2 due to the higher adiabatic negative-ion curve as compared with the results for model 1 . The vibrational Feshbach resonances will be discussed further in the next section.

In Figure 7 we show the attachment rates for each molecule as a function of temperature and compare the rates for $\mathrm{CH}_{3} \mathrm{I}$ with experimental measurements. Again the rate of increase of the 


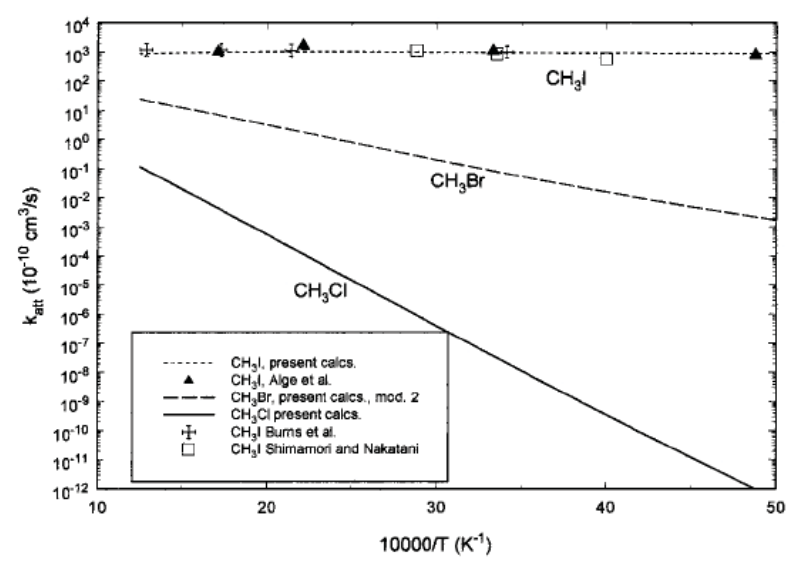

Figure 7. Arrhenius type plot of the calculated attachment rates for all methyl halides studied here, present calculations and experimental data for $\mathrm{CH}_{3} \mathrm{I}$ of Alge et al [11], Burns et al [16] and Shimamori and Nakatani [21].

attachment rates is also connected with the position of the crossing point. As the crossing point moves to lower energy, from $\mathrm{CH}_{3} \mathrm{Cl}$ to $\mathrm{CH}_{3} \mathrm{Br}$ to $\mathrm{CH}_{3} \mathrm{I}$, the attachment rates vary more slowly with temperature. The activation energy for $\mathrm{CH}_{3} \mathrm{Cl}$ from the present calculations is $611 \mathrm{meV}$. We do not show any experimental measurements in Figure 7. The swarm measurements of [3] determined a value on the order of $10^{-13} \mathrm{~cm}^{3} \mathrm{~s}^{-1}$. The measurements of [13] determined an upper limit for the attachment rate to $\mathrm{CH}_{3} \mathrm{Cl}$ at thermal electron energies of $1.9 \times 10^{-15} \mathrm{~cm}^{3} \mathrm{~s}^{-1}$. Both of these values are much larger than our calculations at room temperature. Datskos et al [1] have measured the attachment rates as a function of the mean electron energy in a swarm experiment for temperatures in the range $300-750 \mathrm{~K}$. These measurements show an increase of the rate by three to four orders of magnitude over this temperature range. At $300 \mathrm{~K}$ these measurements obtain a value on the order of $10^{-14} \mathrm{~cm}^{3} \mathrm{~s}^{-1}$ and a value near $10^{-10} \mathrm{~cm}^{3} \mathrm{~s}^{-1}$ at $750 \mathrm{~K}$. These values are somewhat larger than the present calculations, but such a large temperature dependence seems consistent with our calculations. We are unaware of any measurements of the temperature dependence of the thermal attachment rate for $\mathrm{CH}_{3} \mathrm{Cl}$.

In the case of $\mathrm{CH}_{3} \mathrm{I}$ the attachment rates for temperatures above $500 \mathrm{~K}$ start to decrease and do not obey equation (6). A similar decrease for higher temperatures was observed in the measurements of [11], though the uncertainties in the measurements at high temperatures for $\mathrm{CH}_{3} \mathrm{I}$ are quite large. The activation energy of $25 \mathrm{meV}$ obtained in [11] for $\mathrm{CH}_{3} \mathrm{I}$ is only for temperatures at or below $452 \mathrm{~K}$. We are unable to obtain a good fit to equation (8) for temperatures in the range 450 down to $300 \mathrm{~K}$. We also show the measurements for $\mathrm{CH}_{3} \mathrm{I}$ of Burns et al [16] and of Shimamori and Nakatani [21].

In Figure 8 we show attachment rates for $\mathrm{CH}_{3} \mathrm{I}$ from temperatures lower than $200 \mathrm{~K}$ up to 800 $\mathrm{K}$ for several gas temperatures. At low temperatures the rate rises with a $T_{\mathrm{e}}^{-1 / 2}$ dependence on temperature. This is due to the dominance of the $v=0$ vibrational level in the temperature average at low temperatures and the $1 / E^{x}$ dependence of the DEA cross sections on electron energy at low energies. This rise in the attachment rate at low electron temperatures has been observed in [24]. Recent measurements by Speck et al [39] agree with the calculations in Figure 8 near room temperature, but at low temperatures these measurements decrease. The reason for this discrepancy is not clear at this time. 


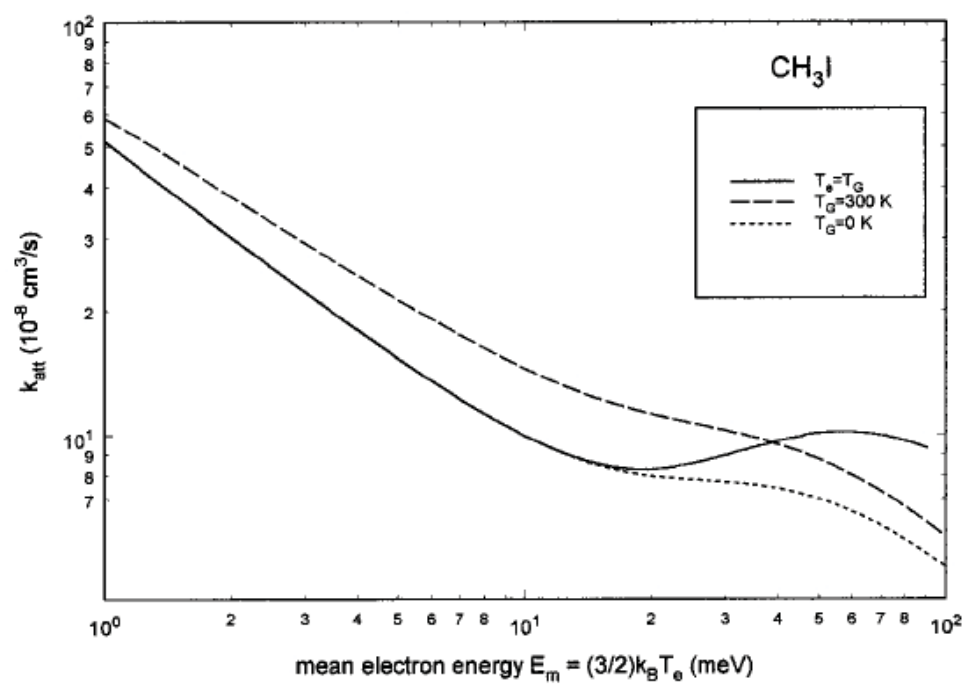

Figure 8. Logarithmic plot of the attachment rates for $\mathrm{CH}_{3} \mathrm{I}$ from $5 \mathrm{~K}$ up to $800 \mathrm{~K}$ as a function of the mean electron energy, $E_{\mathrm{m}}$, for several gas (vibrational) temperatures. $T_{\mathrm{e}}=T_{\mathrm{G}}$ means that the electron temperature equals the gas temperature, $T_{\mathrm{G}}=300 \mathrm{~K}$ or $T_{\mathrm{G}}=0 \mathrm{~K}$ that the gas temperature is kept at a constant value of 300 or $0 \mathrm{~K}$.

Table 2. Activation energies, $E_{\mathrm{a}}$ (in $\mathrm{meV}$ ), of the temperature-averaged DA cross sections at several electron energies, $E$, for $\mathrm{CH}_{3} \mathrm{Cl}$ and $\mathrm{CH}_{3} \mathrm{Br}$.

\begin{tabular}{llll}
\hline Electron energy $(\mathrm{eV})$ & $E_{\mathrm{a}}(E), \mathrm{CH}_{3} \mathrm{Cl}$ & $E_{\mathrm{a}}(E), \mathrm{CH}_{3} \mathrm{Br}$ model 1 & $E_{\mathrm{a}}(E), \mathrm{CH}_{3} \mathrm{Br}$ model 2 \\
\hline 0.001 & 658 & 291 & 298 \\
0.01 & 652 & 286 & 295 \\
0.05 & 615 & 264 & 262 \\
0.1 & 572 & 231 & 225 \\
0.2 & 489 & 174 & 144 \\
\hline
\end{tabular}

It is interesting that the attachment rates of the molecules $\mathrm{CH}_{3} \mathrm{Cl}$ and $\mathrm{CH}_{3} \mathrm{Br}$ obey the simple equation (8). This, so-called, Arrhenius type of dependence of the DEA cross sections and attachment rate was first studied for $\mathrm{O}_{2}$ [40]. We also note that the temperature-averaged DEA cross sections for these molecules have the same form as equation (8). In Table 2 we show the parameters of equation (8), which we have fitted to DEA cross sections of both molecules for several electron energies. The temperature-averaged cross sections also obey the Arrhenius law and the slope (activation energy) decreases as the electron energy increases.

It is well known that the DEA cross sections from individual vibrational levels rise dramatically, in the molecules $\mathrm{Na}_{2}$ and $\mathrm{CF}_{3} \mathrm{Cl}$, for example, for levels just below the energy of the crossing point $[30,41]$. This type of behavior has generally been attributed to the fact that the range of internuclear distance, $\rho$, from which the vertical transition to the anion curve may take place becomes wider for higher vibrational levels [42]. The transition thus may take place closer to the crossing point, making the survival probability larger. For vibrational levels above the crossing point energy (exoergic levels) the individual DEA cross sections are known to stagnate or even to decrease.

It is also known that the Arrhenius behavior of the attachment rate arises when the cross section vanishes below a threshold [43]. The behavior of the DEA cross sections from individual vi- 
brational levels described in the preceding paragraph approximates this step function type of behavior. The temperature-averaged DEA cross section is given by

$$
\sigma(E, T)=\frac{\sum_{v} \sigma_{v}(E) \exp \left[-\frac{E_{v}}{T}\right]}{\sum_{v} \exp \left[-\frac{E_{v}}{T}\right]} .
$$

If we assume the DEA cross sections below a certain level, $v^{*}$, are negligible and take only the largest terms in both sums of equation (9) we obtain the Arrhenius type of behavior with the activation energy given by

$$
E_{\mathrm{a}}=E_{v^{*}}-E_{0},
$$

where $E_{0}$ is the energy of the ground vibrational level. Alternatively, if the vibrational levels are closely spaced one can use the harmonic approximation for $E_{\mathrm{v}}$ and convert the sums in equation (9) to an integral. If the individual DEA cross sections have the step function type of behavior one obtains the exact Arrhenius behavior with the activation energy equal to the energy at which the step occurs.

In the methyl halides studied here, the largest term in the numerator of equation (9) comes from the vibrational level which occurs just above the maximum in the adiabatic negative-ion potential. In $\mathrm{CH}_{3} \mathrm{Cl}$ this level is $v^{*}=8$, in $\mathrm{CH}_{3} \mathrm{Br}$ it is $v^{*}=4$ and in $\mathrm{CH}_{3} \mathrm{I} v^{*}=1$. Then, using equation (10) gives the activation energy for $\mathrm{CH}_{3} \mathrm{Cl}$ of $692 \mathrm{meV}$ and for $\mathrm{CH}_{3} \mathrm{Br} 297 \mathrm{meV}$. These values are in rough agreement with those of Table 2 for the lowest electron energies. This shows that DEA at low energies is dominated by the cross section from the vibrational level which occurs just above the barrier created by the adiabatic negative-ion potential curve. The activation energies of Table 2 decrease with increasing energy since the DEA from the lower vibrational levels becomes more important at higher electron energies.

In the case of $\mathrm{CH}_{3} \mathrm{I}$, using $v^{*}=1$ in equation (10) gives an activation energy of $66 \mathrm{meV}$. However, in $\mathrm{CH}_{3} \mathrm{I}$ the adiabatic barrier occurs at such a low energy that the step-function-like behavior does not occur and one may not expect the Arrhenius behavior to be appropriate in this case. In [11] the authors suggest that the attachment rate for $\mathrm{CH}_{3} \mathrm{I}$ should decrease at higher temperatures since at some point the attachment cross section will reach a maximum value imposed by unitarity. However, it seems to us that this behavior, in this case, is actually due to the low energy of the crossing point. As shown in Figure 8 the attachment rate for $\mathrm{CH}_{3} \mathrm{I}$ also does not have an Arrhenius type of behavior at low temperatures, but in this case the rate increases due to the average over the Maxwellian electron energy distribution.

\section{Vibrational Feshbach resonances}

The vibrational Feshbach resonance is associated with a temporary capture of the incident electron in the field of the vibrationally excited molecule. These resonances were predicted [27] on the basis of the zero-range-potential approximation, but they are more likely supported by a long-range electron-molecule interaction. A vibrational Feshbach resonance in the DEA channel was first observed experimentally in high-resolution measurements [24] of DEA to $\mathrm{CH}_{3}$ I. The resonance appears as a prominent peak in the DEA cross sections just below the $v=1$ threshold. In this case it occurs due to a combined effect of dipolar and polarization interactions. The previous calculations of DEA to $\mathrm{CH}_{3} \mathrm{Cl}[7,8]$ also exhibit peaks in the temperature-averaged cross sections which were explained by dominance of a particular vibrational level in a certain interval of electron energies. However, the energy dependence of each partial cross section was not analyzed in detail, particularly because calculations were not performed with a small enough energy step. Here we present 

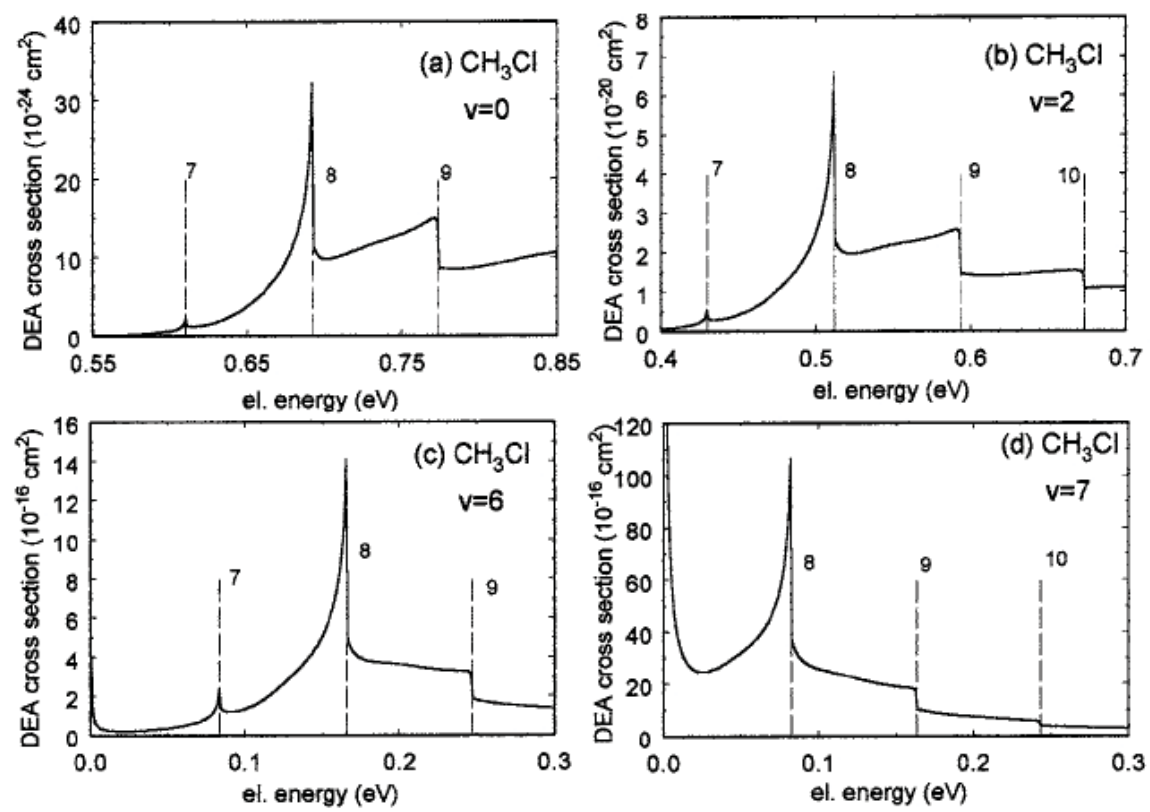

Figure 9. DEA cross sections from individual vibrational levels, $v=0,2,4$ and 7 of $^{\mathrm{CH}_{3} \mathrm{Cl}}$.

more detailed results showing that the structure calculated before for $\mathrm{CH}_{3} \mathrm{Cl}$ is also associated with vibrational Feshbach resonances.

In Figure 9 we present cross sections for DEA from different vibrational states of $\mathrm{CH}_{3} \mathrm{Cl}$. All cross sections exhibit a pronounced peak below the $v=8$ threshold and a relatively weak structure below the $v=7$ threshold. Cross sections at all other thresholds exhibit cusps. As in the case of $\mathrm{CH}_{3} \mathrm{I}$ [24], we can interpret this structure in terms of the potential curves presented in Figures 1 and 6. The adiabatic potential curve for $\mathrm{CH}_{3} \mathrm{Cl}^{-}$follows the neutral curve in the region close to equilibrium internuclear separation producing about eight vibrationally excited states of $\mathrm{CH}_{3} \mathrm{Cl}^{-}$ with a loosely bound electron. In the case of $\mathrm{CH}_{3} \mathrm{I}$ we have only one vibrationally excited state of this type, therefore the resonance occurs only at the $v=1$ threshold. For $\mathrm{CH}_{3} \mathrm{Cl}$ we have eight such states. However, for the thresholds with $v<7$ resonances are masked by a rapid increase of the DEA cross section due to the very fast growth of the Franck-Condon overlap between the initial vibrational state and the dissociating state. Note that the $v=8$ threshold corresponds to different electron energies for different initial vibrational states $v_{\mathrm{i}}$. Therefore one peak in each partial cross section produces several peaks in the temperature-averaged cross sections. The peak with the highest energy in this series, $E=0.68 \mathrm{eV}$, corresponds to the $v_{\mathrm{i}}=0$ state. Moving towards lower energies we find the peaks corresponding to higher values of $v_{i}$. Note, however, that the magnitude of the peak varies enormously with $v_{\mathrm{i}}$ being about $10^{-7} \AA^{2}$ for $v_{\mathrm{i}}=0$ and about $1 \AA^{2}$ for $v_{\mathrm{i}}=5$. Therefore at higher temperatures peaks corresponding to low $v_{\mathrm{i}}$ are masked by the large background due to higher $v_{\mathrm{i}}$.

A similar picture is observed for $\mathrm{CH}_{3} \mathrm{Br}$. Here the adiabatic negative-ion curve of model 2 supports four vibrational states. Due to the lower diabatic negative-ion curve in the region of the crossing point, we observe two vibrational Feshbach resonances at $v=3, v=4$ and thresholds (see Figure 3). 


\section{Conclusions}

We have presented model calculations of DEA to several methyl halides using the resonant $R$-matrix method. For the molecule $\mathrm{CH}_{3} \mathrm{Br}$, the empirical parameters of the theory were first chosen to reproduce the DEA cross sections at $300 \mathrm{~K}$, which were determined by an unfolding procedure from the attachment rates measured in a swarm experiment [9]. The attachment rate calculated using these parameters is seen to be smaller than the experimental measurements. This seems to indicate that a peak in the low-energy DEA cross section would be needed to give such a large attachment rate as measured in [9]. This may perhaps indicate an impurity in the measurement or an inadequacy of the swarm-unfolding procedure at low electron energies. Secondly, we have used parameters which have been obtained by fitting the adiabatic negative-ion curve to ab initio calculations at the MP2 level. In both models the magnitude of the DEA cross section and the temperature dependence of the attachment rates are quite similar. This is due to the similarity of the crossing point of the neutral and anion curves for both models. Model 2, however, has a larger value of the reduced width in the region $\rho>\rho_{\mathrm{c}}$, and thus an adiabatic curve which is substantially different from the diabatic curve. This creates a larger DEA cross section at low electron energies and significantly enhances the vibrational Feshbach resonance which appears just below the $v=4$ vibrational threshold in $\mathrm{CH}_{3} \mathrm{Br}$. We note that the activation energies obtained from both models 1 and 2 are in rather good agreement with the experimental measurements.

We have compared the attachment rates and DEA cross sections for the methyl halides studied here in section 3. Although these molecules can be treated by assuming that the low-energy electron scattering is dominated by a resonance which excites mainly the $v_{3}(\mathrm{C}-\mathrm{Cl})$ symmetric stretching mode, the attachment rates and DEA cross sections vary over a huge range. As we have seen, this is due mainly to the difference in the location of the crossing point in these molecules. As the crossing point moves to lower energy, from $\mathrm{CH}_{3} \mathrm{Cl}$ to $\mathrm{CH}_{3} \mathrm{Br}$ down to $\mathrm{CH}_{3} \mathrm{I}$ the DEA cross sections and attachment rate at room temperature rise dramatically. Also the location of the crossing point determines the activation energy since the DEA cross sections from vibrational levels below this energy rise dramatically with increasing $v$. In the case of $\mathrm{CH}_{3} \mathrm{I}$ our calculated attachment rates do not obey the Arrhenius law and in fact start to decrease for high enough temperatures. In this molecule the crossing point is at such a low energy that this large increase in individual DEA cross sections does not occur.

We have also seen that the agreement with experimental values obtained in the above calculations can only be produced by very accurate $a b$ initio calculations of the adiabatic anion potential curve at the MP2 level for $\mathrm{CH}_{3} \mathrm{Br}$ and $\mathrm{CH}_{3} \mathrm{I}$. In particular, the inclusion of the van der Waals terms in the MP2 method seems to be crucial for calculating accurately the location of the crossing point of the neutral- and negative-ion potential curves.

Finally, we have shown that the structure observed near vibrational thresholds in $\mathrm{CH}_{3} \mathrm{I}$ [24] is probably not unique to this molecule, but is seen in all methyl halides studied here. The strongest feature occurs near the threshold corresponding to the vibrational level which occurs immediately below the crossing point. The features at thresholds below the crossing point energy are interpreted as being due to a vibrationally excited Feshbach resonance created by the long-range dipole plus polarization potential. These features are very sensitive to the width and the adiabatic curve in the region $\rho>\rho_{\mathrm{c}}$.

\section{Acknowledgments}

The authors are indebted to Professors H. Hotop and J. B. A. Mitchell for stimulating discussions on attachment to $\mathrm{CH}_{3}$ I. This work was supported by the NSF through grant PHY-9801871. 


\section{References}

[1] Datskos P G, Christophorou L G, and Carter J G 1990 Chem. Phys. Lett. 168324

[2] Chu S C and Burrow P D 1990 Chem. Phys. Lett. 17217

[3] Petrovic Z Lj, Wang W C, and Lee L C 1989 J. Chem. Phys. 903145

[4] Srivastava S K and Orient O J 1984 Proc. 3rd Int. Symp. on Production and Neutralization of Negative Ions and Beams (Brookhaven) ed K Prelac (New York: AIP)

[5] Pearl D M and Burrow P D 1993 Chem. Phys. Lett. 206483

[6] Pearl D M, Burrow P D, Fabrikant I I, and Gallup G A 1995 J. Chem. Phys. 1159876

[7] Fabrikant I I 1991 J. Phys. B: At. Mol. Opt. Phys. 242213

[8] Fabrikant I I 1994 J. Phys. B: At. Mol. Opt. Phys. 274325

[9] Datskos P G, Christophorou L G, and Carter J G 1992 J. Chem. Phys. 979031

[10] Petrovic Z Lj and Crompton R W 1987 J. Phys. B: At. Mol. Phys. 205557

[11] Alge E, Adams N G, and Smith D 1984 J. Phys. B: At. Mol. Phys. 173827

[12] Mothes K G, Schultes E, and Schindler R N 1972 J. Phys. Chem. 763758

[13] Bansal K M and Fessenden R W 1972 Chem. Phys. Lett. 1521

[14] Wentworth W E, George R, and Keith H 1969 J. Chem. Phys. 511791

[15] Szamrej I, Kosc H, Forys B M, Zytomirski B, and Dzantijew B G 1991 Radiat. Phys. Chem. 38541

[16] Burns S J, Matthews J M, and McFadden D L 1996 J. Phys. Chem. 10019436

[17] Measurements of Lee T G 1963 J. Phys. Chem. 67360 analyzed by Blaunstein R P and Christophorou L G 1968 J. Chem. Phys. 491529

[18] Christodoulides A A and Christophorou L G 1971 J. Chem. Phys. 544691

[19] Spence D and Schulz G J 1973 J. Chem. Phys. 581800

[20] Christophorou L G 1976 Chem. Rev. 76409

[21] Shimamori H and Nakatani Y 1988 Chem. Phys. Lett. 150109

[22] Shimamori H, Tatsumi Y, Ogawa Y, and Sunagawa T 1992 Chem. Phys. Lett. 194223

[23] Alajajian S H, Bernius M T, and Chutjian A 1988 J. Phys. B: At. Mol. Opt. Phys. 214021

[24] Schramm A, Fabrikant I I, Weber J M, Leber E, Ruf M-W, and Hotop H 1999 J. Phys. B: At. Mol. Opt. Phys. 322153

[25] Hotop H, Klar D, Kreil J, Ruf M-W, Schramm A, and Weber J M 1995 The Physics of Electronic and Atomic Collisions ed L J Dubé, J B A Mitchell, J W McConkey, and C E Brion (New York: AIP) p 267

[26] Bardsley N and Mandl F 1968 Rep. Prog. Phys. 31471

[27] Gauyacq J P and Herzenberg A 1982 Phys. Rev. A 252959

[28] Baz’ A I 1957 Zh. Eksp. Teor. Fiz. 33923 (Engl. transl. 1958 Sov. Phys.-JETP 6 709)

[29] Modelli A, Scagnolari F, Distefano G, Jones D, and Guerra M 1992 J. Chem. Phys. 962061

[30] Wilde R S, Gallup G A, and Fabrikant I I 1999 J. Phys. B: At. Mol. Opt. Phys. 32663

[31] Domcke W 1991 Phys. Rep. 20897

[32] Herzberg G 1966 Electronic Spectra of Polyatomic Molecules (Princeton, NJ: Van Nostrand-Reinhold)

[33] Schmidt M W et al 1993 J. Comput. Chem. 141347

[34] Huzinaga S (ed) 1984 Gaussian Basis Sets for Molecular Calculations (New York: Elsevier)

[35] Radzig A A and Smirnov B M 1985 Reference Data on Atoms and Molecules (Berlin: Springer)

[36] Falcetta M and Jordan K D 1990 J. Phys. Chem. 945666

[37] Burrow P D, Modelli A, Chiu N S, and Jordan K D 1982 J. Chem. Phys. 772699

[38] Shi X, Chan V K, Gallup G A, and Burrow P D 1966 J. Chem. Phys. 1041855 Shi X 1992 PhD Thesis University of Nebraska

[39] Speck T, Mostefaoui T, Rebrion-Rowe C, Mitchell J B A, and Rowe B R 2000 J. Phys. B: At. Mol. Opt. Phys. 333575

[40] O’Malley T F 1967 Phys. Rev. 15559

[41] Kulz M, Kortyna A, Keil M, Schellhaass B, and Bergmann K 1993 Phys. Rev. A 48 R4015 Kulz M, Keil M, Kortyna A, Schellhaass B, Hauck J, Bergmann K, and Weyh D 1996 Phys. Rev. A 533324

[42] Wadehra J M 1983 Phys. Rev. A 29 106; Wadehra J M 1989 Phys. Rev. A 413607

[43] Child M S 1974 Molecular Collision Theory (New York: Academic) 